\title{
COMPARATIVE STUDIES OF THE INHIBITORY PROPERTIES OF ANTIBIOTICS ON HUMAN IMMUNODEFICIENCY VIRUS AND AVIAN MYELOBLASTOSIS VIRUS REVERSE TRANSCRIPTASES AND CELLULAR DNA POLYMERASES
}

\author{
Yukinori TAKe, Yoshio InOUYe and Shoshiro NaKAmura \\ Institute of Pharmaceutical Sciences, Hiroshima University School of Medicine, \\ 1-2-3 Kasumi, Minami-ku, Hiroshima 734, Japan
}

\section{H. S. AllaudeEN}

Smith Kline \& French Laboratories, Research and Development Division, P.O. Box 1539, King of Prussia, PA 19406-0939, U.S.A.

\author{
AKINORI KuBo \\ Meiji College of Pharmacy, \\ 1-35-23 Nozawa, Setagaya-ku, Tokyo 154, Japan
}

(Received for publication July 11, 1988)

\begin{abstract}
The inhibition of human immunodeficiency virus (HIV) reverse transcriptase by certain antibiotics and related compounds was studied in comparison with that of avian myeloblastosis virus (AMV) reverse transcriptase and cellular DNA polymerases $\alpha$ and $\beta$. In general, compounds that inhibited HIV reverse transcriptase also inhibited AMV reverse transcriptase. For example, $10 \mu \mathrm{g} / \mathrm{ml}$ of the isoquinoline quinones used in this study inhibited approximately $80 \%$ of the activity of reverse transcriptases of HIV and AMV, but did not inhibit the activity of DNA polymerases $\alpha$ and $\beta$ even at $50 \mu \mathrm{g} / \mathrm{ml}$. AMV enzyme was more sensitive than HIV enzyme to colistin, enduracidins $\mathbf{A}$ and $\mathbf{B}$, janiemycin, glysperin $\mathbf{A}$, and thielavins $\mathbf{A}$ and $\mathbf{B}$. The streptonigrin alkyl esters, however, inhibited HIV reverse transcriptase only. Sakyomicin A, luzopeptins, ellagic acid and suramine inhibited the activities of reverse transcriptases and cellular DNA polymerases.
\end{abstract}

Reverse transcription, a step catalyzed by the unique DNA polymerase, reverse transcriptase, is a pivotal step in the replication of retroviruses and in the stable inheritance of viral genome. An effective inhibitor of reverse transcriptase may selectively block viral replication and therefore, the enzyme has been one of the molecular targets in antiviral studies for many years. We have undertaken a search for inhibitors of avian myeloblastosis virus (AMV) reverse transcriptase among antibiotics and related compounds. Human immunodeficiency virus (HIV), a causative agent for acquired immune deficiency syndrome (AIDS), like other retroviruses, possesses reverse transcriptase. The recent development of recombinant DNA techniques made large amounts of HIV reverse transcriptase available. We wished to compare the characteristics of HIV and AMV reverse transcriptases from the viewpoint of susceptibility to the inhibitors. The selectivity of inhibitors was further studied among DNA polymerases including DNA polymerases $\alpha$ and $\beta$, because many properties of cellular DNA polymerases are shared by reverse transcriptase.

\section{Materials and Methods}

Materials

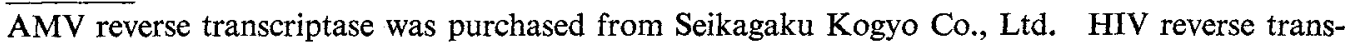


criptase was isolated using the recombinant DNA method ${ }^{1)}$. The coding sequence for the "pol" region of HIV I was inserted into a derivative of the bacterial PAS1 expression vector. The enzyme expressed in Escherichia coli was purified using affinity chromatography ${ }^{1}$.

DNA polymerases $\alpha$ and $\beta$ were isolated from leukocytes of a patient with acute myelogeneous leukemia. They were purified by successive chromatography using DEAE-cellulose, phosphocellulose and single-stranded DNA-cellulose columns according to the procedures described previously ${ }^{2,3}$. The purified enzymes were free of cross contamination and resemble respective viral and cellular DNA polymerases in that they displayed normal preferences of template/primer and monovalent and divalent cations, and sensitivities to certain inhibitors. Poly(dA) and poly $(\mathrm{rA})$ were purchased from Sigma Chemical Company. Oligo(dT $)_{12-18}$ was obtained from Pharmacia Fine Chemicals Co. [Methyl${ }^{3}$ H]TTP was a product of New England Nuclear. All other chemicals were commercial products of the analytical grade.

\section{Assay Methods}

The detailed assay methods for DNA polymerases $\alpha$ and $\beta$ were described by ALLAUDeen et al. ${ }^{2)}$, and AllaudeEN and BerTINo ${ }^{32}$. AMV reverse transcriptase was assayed by the previous method ${ }^{4,5}$ '. Assay of HIV reverse transcriptase was carried out employing the reaction conditions reported by HoFFMAN et al. ${ }^{6)}$ with some modifications ${ }^{7)}$. Briefly, a reaction mixture $(100 \mu l)$ consisting of Tris$\mathrm{HCl} 50 \mathrm{~mm}$ (pH 7.8), dithiothreitol (DTT) $5 \mathrm{~mm}, \mathrm{MgCl}_{2} 5 \mathrm{~mm}, \mathrm{KCl} 150 \mathrm{~mm}$, Triton X-100 $0.05 \%$, glutathione $0.3 \mathrm{mM}$, ethylene glycol bis(2-aminoethyl ether) tetraacetic acid (EGTA) $0.5 \mathrm{~mm}$, poly(rA) $50 \mu \mathrm{g} / \mathrm{ml}$, oligo $(\mathrm{dT})_{12-18} 12 \mu \mathrm{g} / \mathrm{ml},\left[{ }^{3} \mathrm{H}\right] \mathrm{TTP} 10.0 \mu \mathrm{M}(4.8 \mathrm{Ci} / \mathrm{mmol})$ and enzyme was incubated at $37^{\circ} \mathrm{C}$ for 60 minutes. Acid insoluble fractions were collected on a MF-Millipore filter paper (HA, $0.45 \mu \mathrm{m}$ ), washed several times with $5 \%$ TCA containing $2 \mathrm{~mm}$ sodium pyrophosphate and once with $70 \%$ ethanol and air dried. The radioactivity remaining on the filter paper was determined in a liquid scintillation counter.

\section{Results}

The results are shown in Tables 1 to 5 , in which the inhibition exceeding $70 \%$ at the high dose (50 or $40 \mu \mathrm{g} / \mathrm{ml})$ and $/$ or $50 \%$ at the low dose $(10 \mu \mathrm{g} / \mathrm{ml})$ was defined to be significant. In a primary screening using $\mathrm{AMV}$ reverse transcriptase, those compounds listed in Table 1 did not show significant inhibition. Based on the inhibitory activities against HIV reverse transcriptase and DNA polymerases $\alpha$ and $\beta$, the compounds are further classified into three categories as follows; 1) selective inhibitors of AMV reverse transcriptase (Table 2), 2) compounds which inhibit both AMV and HIV reverse transcriptases without affecting cellular DNA polymerases (Table 3 ), and 3) non-specific inhibitors (Table 4).

Streptonigrin and luzopeptins A, B and C are among the most potent inhibitors of AMV reverse transcriptase. Previously, we observed that the streptonigrin $\mathrm{C}-2^{\prime}$ amide derivatives such as the glycine derivative (STN-7) inhibited AMV reverse transcriptase to the same extent as streptonigrin $^{4)}$, whereas streptonigrin methyl

Fig. 1. Structures of streptonigrin derivatives.<smiles>[R]Oc1ccc(-c2c(N)c(-c3ccc4c(n3)C(=O)C(N)=C(O)C4=O)nc(C([CH])=O)c2O)c(O)c1O</smiles>

$\begin{array}{ll}\text { Streptonigrin } & \mathrm{R}=\mathrm{OH} \\ \text { STN-1 } & \mathrm{R}=\mathrm{OCH}_{3} \\ \text { STN-2 } & \mathrm{R}=\mathrm{O}\left(\mathrm{CH}_{2}\right)_{2} \mathrm{CH}_{3} \\ \text { STN-3 } & \mathrm{R}=\mathrm{OCH}\left(\mathrm{CH}_{3}\right)_{2} \\ \text { STN-4 } & \mathrm{R}=\mathrm{O}\left(\mathrm{CH}_{2}\right)_{3} \mathrm{CH}_{3} \\ \text { STN-5 } & \mathrm{R}=\mathrm{O}\left(\mathrm{CH}_{2}\right)_{2} \mathrm{~N}^{\mathrm{C}}\left(\mathrm{CH}_{3}\right)_{2} \\ \text { STN-6 } & \mathrm{R}=\mathrm{O}\left(\mathrm{CH}_{2}\right)_{3} \mathrm{~N}\left(\mathrm{CH}_{3}\right)_{2} \\ \text { STN-7 } & \mathrm{R}=\mathrm{NHCH} \mathrm{COOH}_{2} \\ \text { STN-8 } & \mathrm{R}=\mathrm{NHCH}\left(\mathrm{CH}_{3}\right) \mathrm{COOH} \\ \text { STN-9 } & \mathrm{R}=\mathrm{NH}\left(\mathrm{CH}_{2}\right)_{2} \mathrm{~N}\left(\mathrm{CH}_{3}\right)_{2} \\ \text { STN-10 } & \mathrm{R}=\mathrm{NH}\left(\mathrm{CH}_{2}\right)_{3} \mathrm{~N}\left(\mathrm{CH}_{3}\right)_{2}\end{array}$


Fig. 2. Structures of quinoline quinones and indole quinone.<smiles>O=C1C(=O)C2=C=CC=NC2C1=O</smiles>

QQ-1 $\mathrm{R}=\mathrm{H}$

QQ-2 $\mathrm{R}=\mathrm{CH}_{3}$<smiles>[R]C1=C(C)c2ncccc2C(=O)C1=O</smiles>

QQ-3 $\mathrm{R}=\mathrm{H}$

QQ-4 $\mathrm{R}=\mathrm{CH}_{3}$<smiles>[R20]c1nccc2c1C(=O)C(=O)C(O)C2=O</smiles>

QQ-5 $\quad R_{1}=R_{2}=H$

QQ-6 $\quad \mathrm{R}_{1}=\mathrm{H} \quad \mathrm{R}_{2}=\mathrm{CN}$

QQ-7 $\quad \mathrm{R}_{1}=\mathrm{CH}_{3} \quad \mathrm{R}_{2}=\mathrm{H}$

QQ-8 $\quad \mathrm{R}_{1}=\mathrm{CH}_{3} \quad \mathrm{R}_{2}=\mathrm{CN}$

QQ-9 $\mathrm{R}_{1}=\mathrm{R}_{2}=\mathrm{CH}_{3}$

ester (STN-1) did not inhibit the enzyme activity ${ }^{4,8)}$. As well as STN-7, the other streptonigrin amide derivatives, STN-8, 9 and 10, inhibited the reverse transcriptases from AMV and HIV to an equal extent (Table 3). Four of the ester derivatives of streptonigrin, STN-1, 2, 3 and 4, inhibited only the reverse transcriptase of HIV but the other three enzymes were not inhibited by them (Table 5 ). This observation is consistent with the report by Ceririgos et al. ${ }^{87}$ who showed that STN-1 did not inhibit the AMV reverse transcriptase activity. It is worth noting that<smiles>COc1ccncc1C(=O)O</smiles>

QQ-10 $\quad \mathrm{R}=\mathrm{H}$

QQ-11 $\mathrm{R}=\mathrm{CH}_{3}$<smiles>Cc1c(C)c2c(=O)ccc(=O)c1=2</smiles>

IQ-1

the parent compound, streptonigrin, inhibited the activity of AMV reverse transcriptase but not cellular DNA polymerases examined ${ }^{\theta)}$. Although the ester derivatives, STN-1 to STN-4, lacked the ability to inhibit AMV reverse transcriptase, the dimethylamino group endowed the ester derivatives with inhibitory activity against not only AMV reverse transcriptase but also HIV enzyme (see the results for STN-5 and 6 in Table 3). We have included the quinoline quinones in our study because of their structural resemblance to streptonigrin ${ }^{9 \sim 12)}$. As in the case of streptonigrin, they are potent inhibitors of both AMV and HIV reverse transcriptases, but not cellular DNA polymerases (Table 3). For example, the quinones used in this study, at $10 \mu \mathrm{g} / \mathrm{ml}$, inhibited the reverse transcriptases of HIV and AMV approximately $80 \%$; they did not inhibit the DNA polymerases $\alpha$ and $\beta$ activities even at $50 \mu \mathrm{g} / \mathrm{ml}$.

Sakyomicin A showed moderate inhibition of AMV reverse transcriptase ${ }^{13)}$; the inhibition values at 40 and $10 \mu \mathrm{g} / \mathrm{ml}$ were 63 and $26 \%$, respectively. The role of naphthoquinone moiety as the minimum requisite for this activity was confirmed previously ${ }^{14)}$. Besides AMV reverse transcriptase, HIV enzyme was inhibited by sakyomicin A and the naphthoquinone derivatives such as NQ-1, 2, 3 
Table 1. Antibiotics and other compounds not showing significant inhibition of AMV reverse transcriptase.

\begin{tabular}{|c|c|c|}
\hline Peptides & Ezomycins A1 and A2 & DNA Gyrase Inhibitors \\
\hline Actinomycin D & Formycin A & Cumermycin Al \\
\hline Aculeacin A & Herbicidin A & Nalidixic acid ${ }^{a}$ \\
\hline Amidinomycin & Minimycin & Norfloxacin ${ }^{a}$ \\
\hline Bacitracin & Neplanomycins A, B, C, D and F & Noboviocin $^{\mathrm{a}}$ \\
\hline Capreomycin & Nucleocidin & \\
\hline Echinomycin & Oxamicetin & Miscellaneous \\
\hline Empedopeptin & Polyoxins $\mathrm{A}$ and $\mathrm{C}$ & Azomycin A \\
\hline Ilamycin & Tubercidin & Cerexin A \\
\hline Pyridomycin & & Chartreucin \\
\hline Siomycin A & Ansamycins & Chicamycin \\
\hline \multirow[t]{2}{*}{ Triostin A } & Ansamitocin & Chloramphenicol \\
\hline & Geldanamycin & Chromostin \\
\hline Aminoglycosides & Rifampicin & Deoxyfrenolicin \\
\hline Butirosine A & & Glysperins B and C \\
\hline Glebomycin & Macrolides & Histidinomycin \\
\hline Inosamycins $\mathrm{A}, \mathrm{C}$ and $\mathrm{D}$ & 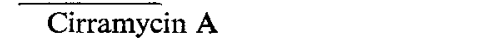 & Isohematinic acid \\
\hline Neomycin B & Erythromycin & Julymycin \\
\hline Paromomycin & Kitasamycin & Lincomycin \\
\hline Sorbistin A1 & & Miharamycin A \\
\hline \multirow[t]{2}{*}{ Xylostatin } & Bleomycins & Mitomycins $\mathrm{A}$ and $\mathrm{C}$ \\
\hline & Bleomycins A2, A5 and B2 & Mycoplanecin \\
\hline Anthracyclines & Peplomycin & Novomycin \\
\hline Aclarubicin & Tallysomycins A and B & Pholipomycin \\
\hline Baumycin & & Pyrrolnitrin \\
\hline Daunorubicin & Polyenes & Showdomycin \\
\hline Figaroic acid & Trichomycin & Streptolydigin \\
\hline \multirow[t]{2}{*}{ Marucellomycin } & & Tomaymycin \\
\hline & Pluramycins & Trindamycin \\
\hline Tetracyclines & $\overline{\text { Neopluramycin }}$ & Vancomycin \\
\hline \multirow[t]{2}{*}{ Chlortetracycline } & Pluramycin & $\alpha$-Naphthoflavone ${ }^{a}$ \\
\hline & Neothramycin & $2^{\prime}$-Hydroxychalcone \\
\hline Nucleosides & & Betulin $^{b}$ \\
\hline$\overline{\text { Amicetin }}$ & Polyethers & D- $(+)$-Catechin $b$ \\
\hline Cytarabine $^{a}$ & Dianemycin & Curucumin ${ }^{b}$ \\
\hline Blasticidin $\mathbf{S}$ & Leusermycin & Glycyrrhizin $^{b}$ \\
\hline Bredinin & Lonomycin A & Ursolic acid ${ }^{b}$ \\
\hline Cadeguomycin & Moyukamycin & \\
\hline
\end{tabular}

${ }^{a}$ Synthetic compounds, ${ }^{b}$ compounds of plant origin.

and 4 (Table 4).

Peptide group antibiotics showed a variety of responses. Colistin, enduracidins $\mathbf{A}$ and $\mathbf{B}$, and janiemycin inhibited AMV reverse transcriptase selectively (Table 2), whereas luzopeptins A, B and $C^{15)}$ inhibited all the enzymes tested potently and non-specifically (Table 4). In spite of structural resemblance to luzopeptins, echinomycin and triostin A were not inhibitory against AMV reverse transcriptase (Table 1).

Suramin as well as ellagic acid inhibited all the four enzymes (Table 4). Doxorubicin ${ }^{18)}$, glysperin A, and thielavins A and B were inhibitory to only AMV reverse transcriptase (Table 2). 
Table 2. Antibiotics and other compounds inhibiting preferentially AMV reverse transcriptase.

\begin{tabular}{|c|c|c|c|c|c|c|c|c|c|c|}
\hline & \multicolumn{10}{|c|}{ Inhibition (\%) } \\
\hline & \multirow{2}{*}{\multicolumn{2}{|c|}{$\frac{\text { DP } \alpha}{\text { poly }(\mathrm{dA}) / \text { oligo }(\mathrm{dT})^{\mathrm{a}}}$}} & \multicolumn{4}{|c|}{$\mathrm{DP} \beta$} & \multirow{2}{*}{\multicolumn{2}{|c|}{$\frac{\text { HIV-RT }}{\text { poly(rA)/oligo(dT) }}$}} & \multirow{2}{*}{\multicolumn{2}{|c|}{$\frac{\text { AMV-RT }}{\text { poly(rA)/oligo(dT }}$}} \\
\hline & & & \multicolumn{2}{|c|}{ poly(dA)/oligo(dT) } & \multicolumn{2}{|c|}{ poly(rA)/oligo(dT) } & & & & \\
\hline & $50^{b}$ & 10 & 50 & 10 & 50 & 10 & 50 & 10 & 40 & 10 \\
\hline Doxorubicin & 31 & 1 & 0 & 6 & 21 & 0 & 27 & 0 & 70 & 30 \\
\hline Colistin & 0 & 0 & 0 & 0 & 0 & 0 & 21 & 17 & 89 & 49 \\
\hline Enduracidin A & 0 & 0 & 0 & 6 & 0 & 0 & 32 & 13 & 67 & 50 \\
\hline Enduracidin B & 0 & 0 & 5 & 1 & 0 & 0 & 27 & 1 & 70 & 28 \\
\hline Glysperin A & 0 & 0 & 6 & 0 & 0 & 0 & 6 & 0 & 74 & 60 \\
\hline Janiemycin & 0 & 0 & 7 & 2 & 0 & 0 & 25 & 0 & 80 & 29 \\
\hline Thielavin A & 24 & 0 & 0 & 0 & 0 & 0 & 13 & 4 & 89 & 56 \\
\hline Thielavin B & 0 & 0 & 0 & 0 & 0 & 0 & 8 & 0 & 80 & 56 \\
\hline 1,4-Benzoquinone ${ }^{c}$ & 78 & 60 & 40 & 0 & 92 & 20 & 15 & 0 & 95 & 30 \\
\hline NQ-5 & 40 & 40 & 17 & 4 & 0 & 0 & 0 & 2 & 85 & 80 \\
\hline Fisetin ${ }^{d}$ & 69 & 43 & 27 & 6 & 89 & 11 & 23 & 10 & 100 & 94 \\
\hline
\end{tabular}

Abbreviations: DP, DNA polymerase; HIV-RT, HIV reverse transcriptase; AMV-RT, AMV reverse transcriptase.

a Template/primer, ${ }^{b}$ concentration $(\mu \mathrm{g} / \mathrm{ml}),{ }^{\circ}$ synthetic compounds, ${ }^{a}$ compound of plant origin. 
Table 3. Antibiotics and other compounds inhibiting preferentially AMV and HIV reverse transcriptases.

\begin{tabular}{|c|c|c|c|c|c|c|c|c|c|c|}
\hline & \multicolumn{10}{|c|}{ Inhibition $(\%)$} \\
\hline & \multicolumn{2}{|c|}{$\mathrm{DP} \alpha$} & \multicolumn{4}{|c|}{$\mathrm{DP} \beta$} & \multirow{2}{*}{\multicolumn{2}{|c|}{$\frac{\text { HIV-RT }}{\text { Poly(rA)/oligo(dT) }}$}} & \multirow{2}{*}{\multicolumn{2}{|c|}{$\frac{\text { AMV-RT }}{\text { Poly }(\mathrm{rA}) / \text { oligo(dT) }}$}} \\
\hline & \multicolumn{2}{|c|}{ Poly $(\mathrm{dA}) /$ oligo $(\mathrm{dT})^{\mathrm{a}}$} & \multicolumn{2}{|c|}{ Poly(dA)/oligo(dT) } & \multicolumn{2}{|c|}{ Poly(rA)/oligo(dT) } & & & & \\
\hline & $50^{b}$ & 10 & 50 & 10 & 50 & 10 & 50 & 10 & $40(20)$ & 10 \\
\hline Streptonigrin & 44 & 27 & 0 & 0 & 29 & 3 & 87 & 81 & 93 & 84 \\
\hline STN-5 & 37 & 35 & 0 & 0 & 6 & 0 & 93 & 83 & 84 & 75 \\
\hline STN-6 & 32 & 38 & 0 & 0 & 8 & 0 & 83 & 84 & 91 & 71 \\
\hline STN-7 & 29 & 28 & 0 & 0 & 0 & 0 & 88 & 86 & 93 & 91 \\
\hline STN-8 & 34 & 23 & 0 & 0 & 16 & 11 & 84 & 79 & (81) & 76 \\
\hline STN-9 & 21 & 26 & 27 & 16 & 17 & 0 & 90 & 88 & (61) & 49 \\
\hline STN-10 & 21 & 21 & 19 & 12 & 12 & 6 & 90 & 88 & (77) & 67 \\
\hline QQ-1 & 43 & 28 & 26 & 7 & 25 & 22 & 92 & 92 & 87 & 85 \\
\hline QQ-2 & 25 & 19 & 4 & 0 & 17 & 8 & 87 & 83 & 81 & 67 \\
\hline QQ-3 & 43 & 33 & 1 & 8 & 48 & 23 & 88 & 88 & 90 & 85 \\
\hline QQ-4 & 38 & 28 & 20 & 3 & 47 & 26 & 89 & 88 & 90 & 89 \\
\hline QQ-5 & 0 & 0 & 3 & 0 & 4 & 0 & 86 & 84 & 85 & 81 \\
\hline QQ-6 & 0 & 0 & 0 & 0 & 11 & 0 & 88 & 87 & 82 & 83 \\
\hline QQ-7 & 0 & 0 & 0 & 0 & 6 & 5 & 93 & 85 & 75 & 60 \\
\hline QQ-8 & 14 & 0 & 0 & 0 & 16 & 23 & 91 & 90 & 84 & 73 \\
\hline QQ-9 & 0 & 0 & 0 & 0 & 13 & 11 & 89 & 67 & 68 & 54 \\
\hline QQ-10 & 14 & 0 & 0 & 0 & 23 & 14 & 89 & 85 & 91 & 77 \\
\hline QQ-11 & 25 & 16 & 0 & 0 & 30 & 25 & 92 & 82 & 92 & 88 \\
\hline Sakyomicin A & 40 & 23 & 7 & 7 & 25 & 18 & 59 & 0 & 63 & 26 \\
\hline NQ-1 & 59 & 41 & 2 & 0 & 34 & 9 & 92 & 89 & 81 & 65 \\
\hline NQ-2 & 56 & 45 & 0 & 0 & 40 & 12 & 95 & 85 & 94 & 82 \\
\hline NQ-3 & 66 & 23 & 0 & 0 & 16 & 0 & 85 & 73 & 76 & 34 \\
\hline NQ-4 & 44 & 32 & 0 & 0 & 16 & 13 & 70 & 30 & 77 & 32 \\
\hline
\end{tabular}

Abbreviations: See Table 2 .

STN-5 STN-10: Streptonigrin derivatives, QQ-1 QQ-11 and NQ-1 NQ-4: synthetic compounds.

a Template/primer, ${ }^{\mathrm{b}}$ concentration $(\mu \mathrm{g} / \mathrm{ml})$. 
Table 4. Antibiotics and other compounds inhibiting DNA polymerases non-specifically.

\begin{tabular}{|c|c|c|c|c|c|c|c|c|c|c|}
\hline & \multicolumn{10}{|c|}{ Inhibition $(\%)$} \\
\hline & \multirow{2}{*}{\multicolumn{2}{|c|}{$\frac{\text { DP } \alpha}{\operatorname{Poly}(\mathrm{dA}) / \text { oligo }(\mathrm{dT})^{\mathrm{a}}}$}} & \multicolumn{4}{|c|}{$\mathrm{DP} \beta$} & \multirow{2}{*}{\multicolumn{2}{|c|}{$\frac{\text { HIV-RT }}{\text { Poly }(\mathrm{rA}) / \text { oligo(dT) }}$}} & \multirow{2}{*}{\multicolumn{2}{|c|}{$\frac{\text { AMV-RT }}{\text { Poly(rA)/oligo(dT }}$}} \\
\hline & & & \multicolumn{2}{|c|}{ Poly(dA)/oligo(dT) } & \multicolumn{2}{|c|}{ Poly(rA)/oligo(dT) } & & & & \\
\hline & $50^{b}$ & 10 & 50 & 10 & 50 & 10 & 50 & 10 & 40 & 10 \\
\hline Luzopeptin A & 93 & 66 & 91 & 62 & 94 & 74 & 85 & 68 & 100 & 89 \\
\hline Luzopeptin B & 100 & - 100 & 100 & 99 & 98 & 98 & 100 & 95 & 96 & 97 \\
\hline Luzopeptin C & 98 & 100 & 100 & 99 & 99 & 99 & 100 & 99 & 100 & 100 \\
\hline Ellagic acid ${ }^{c}$ & 81 & 0 & 59 & 0 & 99 & 30 & 75 & 36 & 94 & 95 \\
\hline Suramin ${ }^{\mathrm{e}}$ & 71 & 31 & 0 & 0 & 98 & 30 & 100 & 84 & 96 & 81 \\
\hline NQ-6 & 22 & 27 & 3 & 0 & 0 & 0 & 0 & 0 & 0 & 5 \\
\hline NQ-7 & 26 & 5 & 22 & 16 & 18 & 9 & 0 & 0 & 0 & 11 \\
\hline NQ-8 & 16 & 6 & 16 & 8 & 7 & 0 & 0 & 0 & 1 & 5 \\
\hline IQ-1d & 40 & 2 & 0 & 0 & 15 & 17 & 22 & 9 & 18 & 0 \\
\hline
\end{tabular}

Abbreviations: See Table 2 .

a Template/primer, ${ }^{b}$ concentration $(\mu \mathrm{g} / \mathrm{ml}),{ }^{c}$ compounds of plant origin, ${ }^{a}$ synthetic compounds.

Table 5. Streptonigrin derivatives inhibiting HIV reverse transcriptase exclusively.

\begin{tabular}{|c|c|c|c|c|c|c|c|c|c|c|}
\hline & \multicolumn{10}{|c|}{ Inhibition $(\%)$} \\
\hline & \multicolumn{2}{|c|}{$\operatorname{DP} \alpha$} & \multicolumn{4}{|c|}{$D P \beta$} & \multirow{2}{*}{\multicolumn{2}{|c|}{$\frac{\text { HIV-RT }}{\text { Poly(rA)/oligo(dT) }}$}} & \multirow{2}{*}{\multicolumn{2}{|c|}{$\frac{\text { AMV-RT }}{\text { Poly }(\mathrm{rA}) / \text { oligo(dT) }}$}} \\
\hline & \multicolumn{2}{|c|}{ Poly(dA)/oligo(dT) ${ }^{a}$} & \multicolumn{2}{|c|}{ Poly $(\mathrm{dA}) /$ oligo(dT) } & \multicolumn{2}{|c|}{ Poly(rA)/oligo(dT) } & & & & \\
\hline & $50^{b}$ & 10 & 50 & 10 & 50 & 10 & 50 & 10 & 40 & 10 \\
\hline STN-1 & 37 & 29 & 22 & 7 & 13 & 0 & 68 & 23 & 12 & 0 \\
\hline STN-2 & 17 & 8 & 18 & 13 & 9 & 8 & 66 & 24 & 18 & 0 \\
\hline STN-3 & 30 & 20 & 17 & 17 & 13 & 11 & 74 & 7 & 18 & 6 \\
\hline STN-4 & 38 & 30 & 0 & 0 & 3 & 0 & 67 & 0 & 0 & 0 \\
\hline
\end{tabular}

Abbreviations: See Table 2.

a Template/primer, ${ }^{b}$ concentration $(\mu \mathrm{g} / \mathrm{ml})$. 


\section{Discussion}

Many compounds used in this study inhibited the reverse transcriptases of HIV and AMV. However, some compounds listed in Table 2 inhibited preferentially AMV reverse transcriptase; the ester derivatives of streptonigrin, on the other hand, inhibited HIV reverse transcriptase only. Such differences between the reverse transcriptases of HIV and AMV and the structure activity relationship data obtained using these compounds may be exploited in designing better and more selective inhibitors.

As for quinone compounds such as streptonigrin and sakyomicin A, we have proposed the existence of a specific site of interaction, referred to as a "quinone pocket", on AMV reverse transcriptase $^{17)}$. On the basis of the results shown in Table 3, we postulate that HIV reverse transcriptase also possesses a "quinone pocket" which is concerned with the sensitivity of this enzyme to quinone compounds of both synthetic and natural origins.

Among the peptide group antibiotics, luzopeptins showed a significant inhibition of HIV reverse transcriptase; however they also inhibited the other enzymes used in this study. The effects of luzopeptin $\mathrm{C}$, janiemycin and colistin on the in vitro replication of HIV have been reported earlier ${ }^{15)}$. The replication of HIV in MT-4 cells was suppressed in the presence of luzopeptin C at the concentrations not affecting cell viability. Meantime, no effect on viral replication and cell viability was observed in the case of the other antibiotics. Which function of luzopeptin $\mathrm{C}$ plays a crucial role in the inhibition of in vitro HIV replication, however, remains to be clarified.

\section{Acknowledgment}

We thank Drs. C. Debouck and T. Francis for their supplying HIV reverse transcriptase and Drs. D. DRUTZ and P. ACTOR for their helpful support. This work was partly supported by a Grant-in-Aid for general scientific research from the Ministry of Education, Science and Culture, Japan.

\section{References}

1) Francis, T.; M. Maquire \& C. Debouck: A targeted screen for natural product inhibitors of HIV reverse transcriptase. in preparation

2) Allaudeen, H. S.; J. Descamps, R. K. Sehgal \& J. J. Fox: Selective inhibition of DNA replication in herpes simplex virus infected cells by 1-(2'-deoxy-2'-fiuoro- $\beta$-D-arabinofuranosyl)-5-iodocytosine. J. Biol. Chem. 257: 11879 11882, 1982

3) Allaudeen, H. S. \& J. R. Bertino: Inhibition of activities of DNA polymerase $\alpha, \beta, \gamma$ and reverse transcriptase of L1210 cells by phosphonoacetic acid. Biochim. Biophys. Acta 520: 490 497, 1978

4) Okada, H.; H. Mukal, Y. Inouye \& S. Nakamura: Biological properties of streptonigrin derivatives. II. Inhibition of reverse transcriptase activity. J. Antibiotics 39: 306 308, 1986

5) Hanajima, S.; K. Ishimaru, K. Sakano, S. K. Roy, Y. Inouye \& S. Nakamura: Inhibition of reverse transcriptase by limocrocin. J. Antibiotics 38: 803 805, 1985

6) Hoffman, A. D.; B. Banapour \& J. A. Levy: Characterization of the AIDS-associated retrovirus reverse transcriptase and optimal conditions for its detection in virions. Virology 147: 326 335, 1985

7) Rey, M. A.; B. Spire, D. Dormont, F. Barre-Sinoussi, L. Montagnier \& J. C. Chermann: Characterization of the RNA dependent DNA polymerase of a new human T lymphotropic retrovirus (lymphoadenopathy associated virus). Biochem. Biophys. Res. Commun. 121: 126 133, 1984

8) Chirigos, M. A.; J. W. Pearson, T.S. Papas, W. A. Woods, H. B. Wood, Jr. \& G. Spahn: Effects of streptonigrin (NSC-45383) and analogs on oncornavirus replication and DNA polymerase activity. Cancer Chemother. Rep. 57: 305 309, 1973

9) Inouye, Y.; Y. TAke, K. Oogose, A. Kubo \& S. Nakamura: The quinoline quinone as the minimum entity for reverse transcriptase inhibitory activity of streptonigrin. J. Antibiotics 40:105 107, 1987

10) Take, Y.; K. Oogose, T. Kubo, Y. Inouye, S. Nakamura, Y. Kitahara \& A. Kubo: Comparative study on biological activities of heterocyclic quinones and streptonigrin. J. Antibiotics 40: 679 684, 1987

11) InOUYe, Y.; K. Oogose, Y. TAKe, T. Kubo \& S. NaKamura: Role of single-electron reduction potential in inhibition of reverse transcriptase by streptonigrin and sakyomicin A. J. Antibiotics 40: 702 705, 1987

12) Oogose, K.; Y. Hafuri, E. Takemori, E. Nakata, Y. Inouye, S. NaKamura \& A. Kubo: Mechanism of inhibition of reverse transcriptase by quinone antibiotics. J. Antibiotics 40:1778 1781, 1987 
13) Tanaka, N.; T. Okabe, N. Tanaka, Y. Take, Y. Inouye, S. Nakamura, H. Nakashima \& N. Yamamoto: Inhibition by sakyomicin $A$ of avian myeloblastosis virus reverse transcriptase and proliferation of AIDSassociated virus (HTLV-II/LAV). Jpn. J. Cancer Res. 77: 324 326, 1986

14) Take, Y.; M. Sawada, H. KunaI, Y. Inouye \& S. Nakamura: Role of the naphthoquinone moiety in the biological activities of sakyomicin A. J. Antibiotics 39:557 563, 1986

15) Inouye, Y.; Y. TAKe, S. Nakamura, H. Nakashima, N. Yamamoto \& H. Kawaguchi: Screening for inhibitors of avian myeloblastosis virus reverse transcriptase and effect on the replication of AIDS-virus. J. Antibiotics 40: 100 104, 1987

16) Nakashima, H.; N. Yamamoto, Y. Inouye \& S. Nakamura: Inhibition by doxorubicin of human immuno-deficiency virus (HIV) infection and replication in vitro. J. Antibiotics 40: 396 399, 1987

17) Hafuri, Y.; E. Takemori, K. Oogose, Y. Inouye, S. Nakamura, Y. Kitahara, S. Nakahara \& A. Kubo: Mechanism of inhibition of reverse transcriptase by quinone antibiotics. II. Dependence on putative quinone pocket on the enzyme molecule. J. Antibiotics 41: 1471 1478, 1988 